\title{
Research of auxiliary analysis function for digital relay protection test
}

\author{
Rui Han ${ }^{a}$,Yufei Teng,Ping Wang,Mingzhong Liu,Yongkang Zheng,Peng
}

\author{
Zhang \\ State Grid Sichuan Electric Power Research Institute, Chengdu 610072, China \\ agdsx110120@126.com
}

\begin{abstract}
Key words: relay protection information; auxiliary analysis; digital relay protection test; smart substation; IEC 61850

Abstract: In order to solve the common problems in digital relay protection test, this paper proposes a digital relay protection test auxiliary analysis function. This function is mainly used to analyze the abnormal situation that occurred during digital relay protection test, and reduce the processing time of abnormal situation, so as to improve the efficiency of digital relay protection test. SCD contains the configuration information of protection device, and the relay protection information can reflect the real-time state and operation state of the relay protection device. Therefore, SCD and relay protection information can be used as the information basis for analyzing testing problems. The proposed function is based on the standardization and unification of relay protection information. Through the analysis of SCD and relay protection information, this function can help testers find out the cause of abnormal situation and put forward some suggestions. The analysis of common problems in digital relay protection test shows that the proposed function can effectively assist testers to handle the abnormal situation during testing process.
\end{abstract}

\section{Introduction}

With the development of smart grid, the construction of smart substation and related technical research have entered rapid development stage. With the increase of smart substations, the test task of digital relay protection devices is more and more arduous. Relay protection device plays an important role in the safe and stable operation of power grid, and its test work is very important.

At present, when conducting the relay protection test, the testers have to set many types of parameters manually, and these parameters need to be reset for different test purposes, which costs a lot of time and makes the efficiency of the testing very low. The low efficiency of the testing is also caused by some other factors. Automatic test is an effective method to improve the test efficiency of relay protection, and has important research significance. At present, there are many researches focusing on automatic testing[1-7]. However, if something abnormal occurs during the automatic testing process, the test result may be invalid, and the testers have to spend a lot of time to find and fix the problem before they restart the testing process.

This paper proposes an auxiliary analysis function for digital relay protection test. Model checking, warning information analysis and testing process analysis are carried out in digital relay protection test based on the analysis of SCD and relay protection information, which will assist testers to deal with the abnormal situation during the testing process, and improve the testing efficiency. Because of standardization and unification of relay protection information, this function is suitable for digital relay protection device from different manufactures. This function can be used 
as an independent module, which can be used in manual testing of digital relay protection and also in the automatic test of digital relay protection.

\section{Introduction of the proposed function}

Whether it's manual testing or automatic testing, problems may be encountered throughout the testing process. The testers are required to find out the cause of the problems and deal with them, and the testing efficiency will be affected.

SCD contains configuration information of the protection device and compares it with the configuration information in the protection device, which makes it possible to check the protection device model. Relay protection information can reflect the real time state and operation condition of the equipment, and plays an important role in equipment condition monitoring and fault diagnosis. The relay protection information can be used for warning information analysis and testing process analysis, and is helpful for the rapid processing of the abnormal condition of the digital relay protection device. At present, the existing standard have standardized and unified the output information of protection devices, so as to meet the advanced application of relay protection such as intelligent diagnosis in smart substations[8].

Therefore, an auxiliary analysis function is proposed. Through the analysis of SCD and relay protection information, it can find out the causes of all kinds of abnormal problems during the test, and provides some suggestions for the testers. The proposed function can be applied to digital relay protection devices from different manufactures with the help of standardization and unification of relay protection information.

\section{Basic information}

The auxiliary analysis function proposed in this paper is mainly based on SCD and relay protection information.

\section{SCD(Substation Configuration Description)}

SCD describes the instance configuration and communication parameters of all IED, the communication configuration between the IED and the primary system structure of the substation[9].

\section{Relay protection information}

The relay protection information used in this paper contains sampled value, protection action, warning information, status change, intermediate node information, device status information etc. Such information can reflect the real-time status and operation condition of the relay protection device.

The detailed classification is as follows:

a) sampled value;

b) protection action: protection action type, protection action phase, protection action time, protection action value;

c) warning information: warning of software and hardware and configuration, link warning, protection warning;

d) status change: GOOSE input, reclosing charge, soft strap, hard strap;

e) intermediate node information: key logical results, critical computation result of protective element

f) device status information: device temperature, operating voltage, light power of optical interface; 
g) protection setting, control word.

The function proposed in this paper will read the relay protection information from the relay protection device through MMS service, which will be used as the data basis of the auxiliary analysis function.

\section{Auxiliary analysis function}

By making full use of SCD and relay protection information, the auxiliary analysis function analyzes the abnormal situation in the digital relay protection test and puts forward some suggestions .

The function mainly consists of three parts:

1.device model checking: at the beginning of the test, comparing the read device configuration information with the configuration information of the device in SCD, which help testers detect the problem of configuration inconsistency timely.

2.warning information analysis: through analysis of the warning information, the warning reason is found out, and treatment advices are provided.

3.testing process analysis: analysis of the test items base on the intermediate node information, etc. can assist testers to locate intermediate node that fail to satisfy the protection logic during the test.

\section{device model checking}

Parse SCD for configuration information, and read device configuration information. By comparing the two types of information above, it can achieve consistency check of setting value, remote measurement, remote signal and strap.

Device model checking is shown in figure 1.

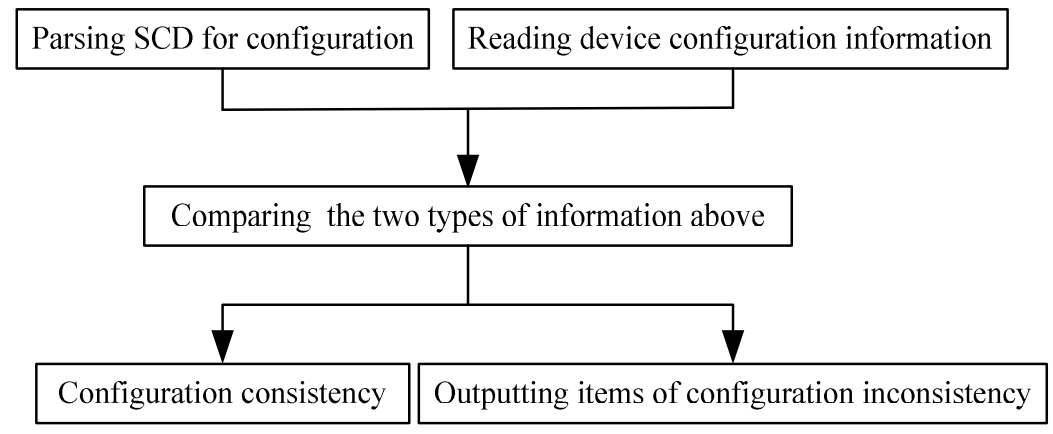

Figure. 1 device model checking

\section{warning information analysis}

During the test, the relay protection information read by MMS service is analyzed to quickly find out the cause of the warning and put forward the treatment advices.

The warning analysis process is shown in figure 2.

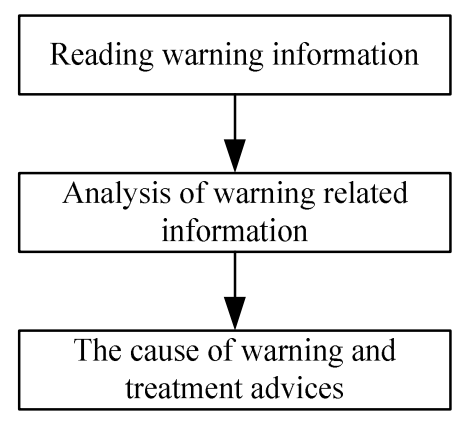

Figure. 2 warning analysis 


\section{testing process analysis}

In the testing process, the parameter setting and the strap setting mainly depend on personal experiences, and the testers may change them frequently, during which they may make mistakes easily. When the test result does not meet the requirements, the sampled value, intermediate node information, and the input are analyzed to check whether the parameters and strap settings are correct and to assist testers to make corresponding corrections.

The analytical method are as follows:

1.Read the sampled value, intermediate node information, input, control word, and strap in the testing process.

2.The protection setting value is determined by the configuration information of the protection device. The values of the control word, strap and input when the protection device acts correctly are determined by the protection logic.

3.Locate node which locks the protection action by comparing the two types of information above.

The analysis process is shown in figure 3.

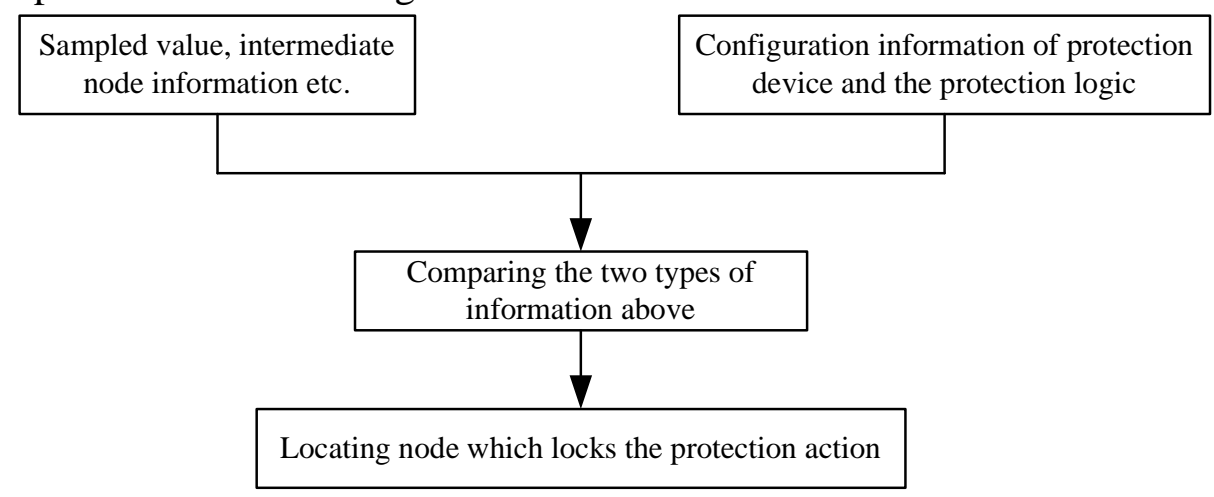

Figure. 3 testing process analysis

\section{Analysis and application}

By using the auxiliary analysis function presented in this paper, two common problems in relay protection test are analyzed.

\section{analysis of optical fiber link interruption}

The digital relay protection device is connected to the merging unit, the intelligent terminal, the switch and other devices through optical fiber. When the protection device emits warning of fiber link interruption, the analysis is as follows:

1.The optical fiber link that have problems can be located through the fiber link warning information.

2.Read the light power of the optical fiber link above, and determine whether it is suitable.

3.If the light power meets the requirements, the testers are suggested to make sure that the output of the tester is valid, and that the optical fiber is in good condition.

Through the analysis above, it can help the testers quickly find out the cause of optical fiber link interruption.

\section{analysis of testing process for bus differential protection}

Bus differential protection is blocked by bus voltage blocking element.

When bus voltage fails to meet the open condition of bus voltage blocking element and the other conditions are met, bus differential protection do not action. The analysis is as follows:

1. Read relevant relay protection information.

a) bus voltage, branch current, differential current; 
b) circuit breaker position;

c) differential protection control word;

d) differential protection soft strap.

2. device configuration information and differential protection logic

a) setting value of differential protection and voltage blocking;

b) circuit breaker needs to be closed;

c) differential protection control word is 1 ;

d) input differential protection soft strap.

3. The comparison of the two types of information above shows that the bus voltage does not meet the requirement of releasing voltage blocking, so bus differential protection fails to operate .

Through the analysis above, the proposed function can help test staff to quickly locate the nodes that do not satisfy the protection logic of bus differential protection.

\section{Conclusion}

In this paper, an auxiliary analysis function during digital relay protection test is proposed to improves the testing efficiency. The proposed function is based on standardization and unification of relay protection information, it can assist the testers to locate the fault points in digital relay protection test, and it can also put forward some suggestions based on analysis of SCD and relay protection information. The proposed function in this paper can be applied to test digital relay protection devices from different manufactures. It can be used as an independent module to assist testers in digital relay protection test.

\section{References}

[1] ZHANG Xiaoli, LIU Huihai, LI Junqing, et al. Automatic Test Platform in Smart Substation for Relay Protection[J]. Automation of Electric Power systems, 2015,39(18): 91-96.

[2] FU Mingjun, LIU Haoyu, DONG Leichao. Research and application of relay protection automatic test system for smart substation[J]. Power System Protection and Control, 2015, 43(1):40-44.

[3] WANG Jun, ZHAN Rongrong. Development of an Auto-test System for the Relay Protection Device[J]. Electrical Automation, 2015, 37(4):93-98.

[4] LAI Qing, HUA Jianwei, LV Yun, et al. Research on general relay protection auto-test system software[J]. Power System Protection and Control, 2010, 38(3):90-94.

[5] WEN Dongxu, YANG Hui, WANG Xuning, et al. Research and practice on the scheme of automatic testing of power protection device security[J]. Power System Protection and Control, 2015, 43(10):135-138.

[6] WANG Zhong, ZHANG Xiaoli, LI Zhongan, et al. Design of automatic test system for relay protection device[J]. Power System Protection and Control, 2015, 43(5):130-135.

[7] DONG Leichao, LIU Haoyu Liu, FU Mingjun, et al. Development of automatic test system for bay level equipment of smart substation[J]. Automation of Electric Power Systems, 2015, 39(5):147-151.

[8] Q/GDW 11010-2013 Information Specification of Protection Relay[S]. Beijing: State Grid Corporation of China, 2014.

[9] Q/GDW 441-2010 Technical Specifications of Protection for Smart Substation[S]. Beijing: State Grid Corporation of China, 2010. 